\title{
Article \\ Application of the Pick Function in the Lieb Concavity Theorem for Deformed Exponentials
}

\author{
Guozeng Yang ${ }^{1}$, Yonggang $\mathrm{Li}^{2, *}$, Jing Wang ${ }^{3}$ and Huafei Sun ${ }^{4,5}$ \\ 1 School of Mathematics and Statistics, Zhengzhou Normal University, Zhengzhou 450000, China; \\ yangguozeng@zznu.edu.cn \\ 2 School of Science, Zhengzhou University of Aeronautics, Zhengzhou 450000, China \\ 3 School of Information, Beijing Wuzi University, Beijing 101149, China; wangjing3@bwu.edu.cn \\ 4 Beijing Institute of Technology, School of Mathematics and Statistics, Beijing 100081, China; \\ huafeisun@bit.edu.cn \\ 5 Yangtze Delta Region Academy of Beijing Institute of Technology, Jiaxing 314000, China \\ * Correspondence: liyonggang914@126.com
}

check for updates

Citation: Yang, G.; Li, Y.; Wang, J.; Sun, H. Application of the Pick Function in the Lieb Concavity Theorem for Deformed Exponentials. Fractal Fract. 2022, 6, 20. https:// doi.org/10.3390/fractalfract6010020

Academic Editor: Savin Treanţă

Received: 18 October 2021 Accepted: 9 December 2021 Published: 31 December 2021

Publisher's Note: MDPI stays neutral with regard to jurisdictional claims in published maps and institutional affiliations.

Copyright: (C) 2021 by the authors. Licensee MDPI, Basel, Switzerland. This article is an open access article distributed under the terms and conditions of the Creative Commons Attribution (CC BY) license (https:// creativecommons.org/licenses/by/ $4.0 /)$.

\begin{abstract}
The Lieb concavity theorem, successfully solved in the Wigner-Yanase-Dyson conjecture, is an important application of matrix concave functions. Recently, the Thompson-Golden theorem, a corollary of the Lieb concavity theorem, was extended to deformed exponentials. Hence, it is worthwhile to study the Lieb concavity theorem for deformed exponentials. In this paper, the Pick function is used to obtain a generalization of the Lieb concavity theorem for deformed exponentials, and some corollaries associated with exterior algebra are obtained.
\end{abstract}

Keywords: Lieb concavity theorem; deformed exponential; Pick function; convexity of matrix

MSC: 15A42; 15A16; 47A56

\section{Introduction}

Matrix theory is widely used in statistics [1], physics [2], computer science [3] and so on. For convenience, $M(n, \mathbb{C})$ is denoted as the set of all $n \times n$ complex matrices ( $\mathbb{C}$ is the set of complex numbers) [4]. $A$ is called a Hermitian matrix when $A \in M(n, \mathbb{C})$ satisfies $A^{*}=A\left(A^{*}\right.$ denotes conjugate transposition of $\left.A\right)$. The Hermitian matrix is frequently used in quadratic forms and their correlation theory [5]. Let $H_{n}$ denote the set of $n \times n$ Hermitian matrices and $H_{n}^{+}$denote the $n \times n$ positive semidefinite Hermitian matrix ( $\mathbb{C}^{n}$ is the $n$ dimensional complex Euclidean space).

Set $u_{1}, u_{2}, \cdots, u_{n}$ to be any orthonormal basis of $\mathbb{C}^{n}$, and then the trace operator $\operatorname{Tr}$ is defined as [4]

$$
\operatorname{Tr}[A]=\sum_{i=1}^{n}\left(u_{i}, A u_{i}\right),
$$

where $(\cdot, \cdot)$ is the inner product of $\mathbb{C}^{n}$. It is well known that for any $A=\left(a_{i j}\right) \in M(n, \mathbb{C})$, the following equalities hold [6]

$$
\operatorname{Tr}[A]=\sum_{j=1}^{n} \lambda_{i}=\sum_{j=1}^{n} a_{i i}
$$

where $\lambda_{i}$ is the eigenvalue of $A$.

From the spectral theorem [5], $A \in H_{n}^{+}$can be decomposed as

$$
A=P^{*} \Lambda_{A} P,
$$


where $P$ is a unitary matrix and $\Lambda_{A}:=\operatorname{diag}\left\{\lambda_{1}, \ldots, \lambda_{n}\right\}$ is a diagonal matrix with eigenvalues $\lambda_{1}, \ldots, \lambda_{n}$. Then, matrix function $f(A)$ is defined as

$$
f(A)=P^{*} f\left(\Lambda_{A}\right) P=\sum_{i=1} f\left(\lambda_{i}\right) P_{i}
$$

where $f\left(\Lambda_{A}\right):=\operatorname{diag}\left\{f\left(\lambda_{1}\right), \ldots, f\left(\lambda_{n}\right)\right\}$ and $P_{i}^{2}=P_{i}$.

Based on the above definition, in 1963, the Wigner-Yanase skew information

$$
\mathrm{I}_{W Y}(\rho)=-\frac{1}{2} \operatorname{Tr}\left[[\sqrt{\rho}, H]^{2}\right]
$$

was introduced by Wigner and Yanase ([7]), where $\rho$ is a density matrix $(\rho \geq 0, \operatorname{tr} \rho=1)$ and $H$ is a Hermitian matrix. Then, an open problem was left

$$
\operatorname{Tr}\left[A^{s} K A^{1-s} K^{*}\right],
$$

which is concave for any positive semidefinite matrix $A$.

In 1973, (2) was proven by Lieb for all $0<s<1$ [8], and a more generalized result was obtained from the following fact [9]

$$
\begin{aligned}
\operatorname{Tr}\left[A^{s} K B^{1-s} K^{*}\right] & =\left\langle K, B^{1-s} K^{*} A^{s}\right\rangle_{\mathcal{L}(H)} \\
& =\left\langle K, \Psi^{-1}\left(B^{1-s} \otimes A^{s}\right) K^{*}\right\rangle_{\mathcal{L}(H)} .
\end{aligned}
$$

where $\Psi^{-1}(A)=\sum_{j}\left(A e_{j}\right) \otimes e_{j}^{*}$. In fact, the Lieb concavity theorem is equivalent to the concavity of $B^{1-s} \otimes A^{s}$.

A more elegant proof of the Lieb concavity theorem appeared in [10] using

$$
\operatorname{Tr}\left[K^{*} A^{s} K B^{1-s}\right]=\left\langle K,\left(A^{s} \otimes B^{1-s}\right) K\right\rangle_{\mathcal{L}(H)},
$$

where

$$
[(A \otimes B) K]_{i, j}=\sum_{k, l} A_{i, k} B_{j, l} K_{k, l} .
$$

In 2009, Effros gave another proof of the Lieb concavity theorem based on the HansenPedersen-Jensen inequality ([11]). Using

$$
L_{A}(K)=A K, R_{B}(K)=K B,
$$

then one obtains

$$
\begin{aligned}
\operatorname{Tr}\left[K^{*} A^{s} K B^{1-s}\right] & =\left\langle K, L_{A^{s}} R_{B^{1-s}}(K)\right\rangle_{\mathcal{L}(H)} \\
& =\left\langle K, R_{B}^{\frac{1}{2}}\left(R_{B}^{-\frac{1}{2}} L_{A} R_{B}^{-\frac{1}{2}}\right)^{s} R_{B}^{\frac{1}{2}}(K)\right\rangle_{\mathcal{L}(H)} .
\end{aligned}
$$

All the above proof of the Lieb concave theorem is equivalent to the joint concavity of commutative operators. In addition, Epstein also obtained the Lieb concave theorem using the theory of Herglotz functions [12].

Recently, Shi and Hansen [13] generalized the Thompson-Golden theorem

$$
\operatorname{Tr}\left[\exp _{q}(A+B)\right] \leq \operatorname{Tr}\left[\left(\exp _{q}(A)\right)^{2-q}\left(A(q-1)+\exp _{q}(B)\right)\right]
$$

As the Thompson-Golden theorem can be regarded as a special form of the Lieb concave theorem, it is worthwhile to study the Lieb concavity theorem for deformed exponentials. In this paper, we will use the theory of the Pick function to obtain a generalization of the Lieb concavity theorem and some other corollaries. The rest of the paper is organized as follows. In Section 2, some general definitions and important conclusions are introduced. 
With these preparations, we obtain some useful results, such as the Lieb concavity theorem, presented in the final Section 3.

\section{Preliminary}

In this section, some general definitions and some important properties are introduced.

\subsection{The q-Logarithm Function and q-Exponential Function}

It is well known that the q-logarithm function $\ln _{q}(x)$ is defined as [13]

$$
\ln _{q}(x)=\left\{\begin{array}{ll}
\frac{x^{q-1}-1}{q-1}, & q \neq 1 \\
\ln x, & q=1
\end{array}\right\}
$$

for any $x>0$. The deformed exponential function or the $q-\operatorname{exponential~} \exp _{q}(x)$ is the inverse function of the $q$-logarithm and is defined as

$$
\exp _{q}(x)=\left\{\begin{array}{cc}
{[(q-1) x+1]^{\frac{1}{q-1}},} & x>\frac{1}{q-1}, \quad q>1 \\
{[(q-1) x+1]^{\frac{1}{q-1}},} & x<\frac{1}{q-1}, \quad q<1 \\
\exp (x), & x \in \mathbb{R}, \quad q=1
\end{array}\right\}
$$

\subsection{Tensor Product and Exterior Algebra}

The tensor product, denoted by " $\otimes$ ", is also called the Kronecker product. It is a generalization of the outer product from vectors to matrices, and the tensor product of matrices is also referred to as the outer product in certain contexts ([9]). For an $m \times n$ matrix $A$ and a $p \times q$ matrix $B$, the tensor product of $A$ and $B$ is defined by

$$
A \otimes B:=\left(\begin{array}{ccc}
a_{11} B & \cdots & a_{1 n} B \\
\vdots & \ddots & \vdots \\
a_{m 1} B & \cdots & a_{m n} B
\end{array}\right)
$$

where $A=\left(a_{i j}\right)_{1 \leq i \leq m, 1 \leq j \leq n}$.

The tensor product is different from matrix multiplication, and one of the differences is commutativity

$$
(I \otimes B)(A \otimes I)=(A \otimes I)(I \otimes B)=A \otimes B .
$$

From the above equations, we obtain

$$
\begin{aligned}
A C \otimes B D & =(A C \otimes I)(I \otimes B D) \\
& =(A \otimes I)(C \otimes I)(I \otimes B)(I \otimes D) \\
& =(A \otimes I)(I \otimes B)(C \otimes I)(I \otimes D) \\
& =(A \otimes B)(C \otimes D) .
\end{aligned}
$$

For convenience, we denote

$$
\otimes_{k} A=\underbrace{A \otimes A \otimes \cdots \otimes A}_{k} .
$$

In addition to the tensor product, there is another common product called exterior algebra [6]. Exterior algebra, denoted by " $\wedge$ ", is a binary operation for any $A_{i} \in H_{n}^{+}$, and the definition is

$$
\begin{aligned}
& (\underbrace{A_{1} \wedge A_{2} \wedge \cdots \wedge A_{k}}_{k})\left(\xi_{i_{1}} \wedge \xi_{i_{2}} \cdots \wedge \xi_{i_{k}}\right)_{1 \leq i_{1}<\cdots<i_{k} \leq n} \\
& =\left(A_{1} \xi_{i_{1}} \wedge A_{2} \xi_{i_{2}} \cdots \wedge A_{k} \xi_{i_{k}}\right)_{1 \leq i_{1}<\cdots<i_{k} \leq n}
\end{aligned}
$$


where $\left\{\xi_{j}\right\}_{j=1}^{n}$ is an orthonormal basis of $\mathbb{C}^{n}$, and

$$
\xi_{i_{1}} \wedge \xi_{i_{2}} \cdots \wedge \xi_{i_{k}}=\frac{1}{\sqrt{n !}} \sum_{\pi \in \sigma_{n}}(-1)^{\pi} \xi_{\pi\left(i_{1}\right)} \otimes \xi_{\pi\left(i_{2}\right)} \cdots \otimes \xi_{\pi\left(i_{k}\right)}
$$

$\sigma_{n}$ is the family of all permutations on $\{1,2, \cdots, n\}$.

Let $\wedge^{k} \mathbb{C}^{n}$ be the span of the $\left\{\xi_{i_{1}} \wedge \xi_{i_{2}} \cdots \wedge \xi_{i_{k}}\right\}_{1 \leq i_{1}<\cdots<i_{k} \leq n}$, and then a simple calculation shows that

$$
\wedge_{n} A=(\underbrace{A \wedge A \wedge \cdots \wedge A}_{k})=\operatorname{det}(A)
$$

\subsection{Pick Function}

Let $z=x+i y$ be a complex number where $i$ is the imaginary unit and $f(z)=$ $U(z)+i V(z)$ is analytic where $U(z), V(z)$ are all real functions. $\operatorname{Re} z=x$ denotes the real part of $z$, and $\operatorname{Im} z=y$ is the imaginary part of $z$. If $\operatorname{Im} f(z)>0$ for any $\operatorname{Im} z>0$, then we call the analytic function $f(z)$ a Pick function [14]. It is equivalent that $f(z)$ is analytic in the upper half-plane with the positive imaginary part.

The Pick functions evidently form a convex cone-for instance, if $\alpha$ and $\beta$ are positive numbers and $f(z)$ and $g(z)$ are two Pick functions, then the function $\alpha f(z)+\beta g(z)$ is also a Pick function. A simple example is that $\tan (z)$ is a Pick function.

$$
\begin{aligned}
\tan (x+i y) & =\frac{\tan (x)+\tan (i y)}{1-\tan (x) \tan (i y)} \\
& =\frac{\tan (x)+i \tanh (y)}{1-i \tan (x) \tanh (y)}
\end{aligned}
$$

Hence, $\operatorname{Im} \tan (z)=\frac{\left(1+\tan ^{2}(x)\right) \tanh (y)}{1+\tan ^{2}(x) \tanh ^{2}(y)}$, and this implies that $\operatorname{Im} \tan (z)>0$ when $y>0$.

It is well known that the Pick function has a integral representation, such as the following lemma [14].

Lemma 1. Let $f(z)$ be a Pick function. Then, $f(z)$ has a unique canonical representation of the form

$$
f(z)=\alpha+\beta z+\int_{R}\left(\frac{1}{\lambda-z}-\frac{\lambda}{1+\lambda^{2}}\right) \mathrm{d} \mu(\lambda),
$$

where $\alpha$ is real, $\beta \geq 0$ and $\mathrm{d} \mu(\lambda)$ is a positive Borel measure on the real $\lambda$-axis that $\int_{R}(1+$ $\left.\lambda^{2}\right)^{-1} \mathrm{~d} \mu(\lambda)$ is finite. Conversely, any function of this form is also a Pick function.

Lemma 1 is frequently used for functions that are positive and harmonic in the halfplane.

\subsection{The Matrix-Monotone Function}

A matrix function $f$ is said to be matrix-monotonic if it satisfies

$$
f(A) \geq f(B) \text { for all } A \geq B>0 .
$$

where $A \geq B>$ is equivalent to $A-B$ is a positive semidefinite Hermitian matrix.

Since the matrix-monotone function is a special kind of operator monotone function, we have the following general conclusions [14].

Lemma 2. The following statements for a real valued continuous function $f$ on $(0,+\infty)$ are equivalent:

(1) $f(z)$ is matrix-monotone;

(2) $f(z)$ admits an analytic continuation to the whole domain $\operatorname{Im} z \neq 0$ and $\operatorname{Im}(z) \operatorname{Im} f(z) \geq 0$. 
(3) $f$ admits an integral representation:

$$
f(\lambda)=\alpha+\beta \lambda+\int_{-\infty}^{0}(1+\lambda t)(t-\lambda)^{-1} \mathrm{~d} \mu(t), \text { for any } \lambda>0
$$

where $\alpha$ is a real number, $\beta$ is non-negative and $\mu$ is a finite positive measure on $(-\infty, 0)$.

From Lemmas 1 and 2, we know that a Pick function must be a matrix-monotone function.

\subsection{Convexity of Matrix}

Suppose that $X$ is a convex set in $\mathbb{R}^{n}$ and $f$ is a function defined on $X$. Then, we call $f$ a convex function if

$$
f\left(t x_{1}+(1-t) x_{2}\right) \leq t f\left(x_{1}\right)+(1-t) f\left(x_{2}\right), \forall x_{1}, x_{2} \in X, \forall t \in[0,1],
$$

for all $x_{1}, x_{2} \in X$ and $t \in[0,1]$.

A matrix function $f$ is called convex if [15-17]

$$
f(t A+(1-t) B) \leq t f(A)+(1-t) f(B),
$$

for any $A, B \in H_{n}^{+}$and any $t \in[0,1]$. Replacing $\leq$ by $<$ in (5), this gives the definition of a strictly matrix convex function. A matrix function $f$ is called (strictly) concave if $-f$ is (strictly) convex. More details can be found in [18].

A matrix convex function must be a convex function; however, the inverse claim is not always true. For instance, the function $f:[0,+\infty) \rightarrow \mathbb{R}$ given by $f(x)=x^{3}$ is a convex function. However, the matrix function $f(A)=A^{3}$ for any $A \in H_{n}^{+}$is not convex.

Let $f(\cdot, \cdot)$ be a bivariate function defined on $H_{n}^{+} \times H_{n}^{+}$. We call $f(\cdot, \cdot)$ jointly convex if

$$
f\left(t A_{1}+(1-t) A_{2}, t B_{1}+(1-t) B_{2}\right) \leq t f\left(A_{1}, B_{1}\right)+(1-t) f\left(A_{2}, B_{2}\right),
$$

for all $A_{1}, A_{2}, B_{1}, B_{2} \in \mathrm{H}_{n}^{+}$and all $t \in[0,1]$.

\subsection{Brunn-Minkowski Inequality}

Finally, let us review the Brunn-Minkowski inequality [19].

Lemma 3. for any $A, B>0$, and then

$$
\left\{\operatorname{Tr}\left[\wedge^{k}(A+B)\right]\right\}^{\frac{1}{k}} \geq\left\{\operatorname{Tr}\left[\wedge^{k} A\right]\right\}^{\frac{1}{k}}+\left\{\operatorname{Tr}\left[\wedge^{k} B\right]\right\}^{\frac{1}{k}}
$$

Proof. Let $\left\{\xi_{i}\right\}_{i=1}^{n}$ be the eigenvectors of $A+B$ with the eigenvalue $\left\{\lambda_{i}\right\}_{i=1}^{n}$, then

$$
\begin{aligned}
\left\{\operatorname{Tr}\left[\wedge^{k}(A+B)\right]\right\}^{\frac{1}{k}} & =\left[\sum_{1 \leq \xi_{i_{1}}<\cdots<\xi_{i_{k}} \leq n} \lambda_{i_{1}} \cdots \lambda_{i_{k}}\right]^{\frac{1}{k}} \\
& =\left[\sum_{1 \leq \xi_{i_{1}}<\cdots<\xi_{i_{k}} \leq n}\left(\operatorname{det}\left|P_{i_{1}, \cdots, i_{k}}^{*}(A+B) P_{i_{1}, \cdots, i_{k}}\right|\right)\right]^{\frac{1}{k}} \\
& \geq\left[\sum_{1 \leq \xi_{i_{1}}<\cdots<\xi_{i_{k}} \leq n}\left(\operatorname{det}\left|P_{i_{1}, \cdots, i_{k}}^{*} A P_{i_{1}, \cdots, i_{k}}\right|+\operatorname{det}\left|P_{i_{1}, \cdots, i_{k}}^{*} B P_{i_{1}, \cdots, i_{k}}\right|\right)\right]^{\frac{1}{k}}
\end{aligned}
$$

where $P_{i_{1}, \cdots, i_{k}}=\left(\xi_{i_{1}}, \cdots, \xi_{i_{k}}\right)$ and $\geq$ holds due to $\operatorname{det}(A+B) \geq \operatorname{det}(A)+\operatorname{det}(B)$. 


$$
\begin{aligned}
& \text { As } S_{k}=\left[\sum_{1 \leq \xi_{i_{1}}<\cdots<\xi_{i_{k}} \leq n} x_{i_{1}} \cdots x_{i_{k}}\right]^{\frac{1}{k}} \text { is concave [20], we have } \\
& \left\{\operatorname{Tr}\left[\wedge^{k}(A+B)\right]\right\}^{\frac{1}{k}} \geq\left[\sum_{1 \leq \xi_{i_{1}}<\cdots<\xi_{i_{k}} \leq n} \operatorname{det}\left|P_{i_{1}, \cdots, i_{k}}^{*} A P_{i_{1}, \cdots, i_{k}}\right|\right]^{\frac{1}{k}} \\
& +\left[\sum_{1 \leq \xi_{i_{1}}<\cdots<\xi_{i_{k}} \leq n} \operatorname{det}\left|P_{i_{1}, \cdots, i_{k}}^{*} B P_{i_{1}, \cdots, i_{k}}\right|\right]^{\frac{1}{k}} \\
& =\left[\sum_{1 \leq \xi_{i_{1}}<\cdots<\xi_{i_{k}} \leq n}\left(\xi_{i_{1}} \wedge \cdots \wedge \xi_{i_{k}}, A \xi_{i_{1}} \wedge \cdots \wedge A \xi_{i_{k}}\right)\right]^{\frac{1}{k}} \\
& +\left[\sum_{1 \leq \xi_{i_{1}}<\cdots<\xi_{i_{k}} \leq n}\left(\xi_{i_{1}} \wedge \cdots \wedge \xi_{i_{k}}, B \xi_{i_{1}} \wedge \cdots \wedge B \xi_{i_{k}}\right)\right]^{\frac{1}{k}} \\
& =\left\{\operatorname{Tr}\left[\wedge^{k} A\right]\right\}^{\frac{1}{k}}+\left\{\operatorname{Tr}\left[\wedge^{k} B\right]\right\}^{\frac{1}{k}} \text {. }
\end{aligned}
$$

\section{Lieb Concavity Theorem for Deformed Exponential}

In this section, we obtain some useful conclusions, and some simple and straightforward computations are omitted. Recently, by using the Young inequality,

$$
\operatorname{Tr}[Y]=\max _{X \geq 0}\left\{\operatorname{Tr}[X]-\operatorname{Tr}\left[X^{2-q}\left(\ln _{q} X-\ln _{q} Y\right)\right]\right\},
$$

Shi and Hansen obtained that $F(A)=\operatorname{Tr}\left[\exp _{q}^{\frac{1}{p}}\left(K^{*} \ln _{q}\left(A^{p}\right) K\right)\right]$ is concave for any $1 \leq q \leq 2$ where $K^{*} K \leq I(I$ is the identity matrix of $M(n, \mathbb{C}))$ [13], namely, the following theorem.

Theorem 1. For $0<p \leq 1,1<q \leq 2$ and $K^{*} K \leq I$, the function

$$
F(A)=\operatorname{Tr}\left[\exp _{q}^{\frac{1}{p}}\left(K^{*} \ln _{q}\left(A^{p}\right) K\right)\right]
$$

is concave for the strictly positive $A \in H_{n}^{+}$.

Proof. (The first proof of Theorem 1)

Since [21]

$$
\mathrm{D} f(A)(B)=\sum_{i} \sum_{j} \frac{f\left(\lambda_{i}\right)-f\left(\lambda_{j}\right)}{\lambda_{i}-\lambda_{i}} P_{i} B P_{j},
$$

we obtain

$$
\begin{aligned}
\frac{\mathrm{d}(\operatorname{Tr}[f(A+t B)-f(A)])}{\mathrm{d} t} & =\operatorname{Tr}\left[\sum_{i} \sum_{j} \frac{f\left(\lambda_{i}\right)-f\left(\lambda_{j}\right)}{\lambda_{i}-\lambda_{i}} P_{i} B P_{j}\right] \\
& =\operatorname{Tr}\left[\sum_{i} P_{j} \sum_{j} \frac{f\left(\lambda_{i}\right)-f\left(\lambda_{j}\right)}{\lambda_{i}-\lambda_{i}} P_{i} B\right] \\
& =\operatorname{Tr}\left[\sum_{i} f^{\prime}\left(\lambda_{i}\right) P_{i} B\right]=\operatorname{Tr}\left[f^{\prime}(A) B\right],
\end{aligned}
$$


where $\lambda_{i}$ are eigenvalues of A. When $f(x)$ is a convex function, we obtain

$$
\operatorname{Tr}[f(A+t B)-f(A)] \geq \operatorname{Tr}\left[f^{\prime}(A) t B\right]
$$

for any $t$. This implies that

$$
\operatorname{Tr}[f(C)]=\max \left\{\operatorname{Tr}\left[f(D)+f^{\prime}(D)(C-D)\right]: D>0\right\} .
$$

Therefore, we obtain

$$
\begin{aligned}
& \operatorname{Tr}\left[\left(K^{*} A^{p q-p} K+I-K^{*} K\right)^{\frac{1}{p q-p}}\right] \\
& =\max \left\{\operatorname{Tr}\left[D^{\frac{1}{p q-p}}+\frac{D^{\frac{1}{p q-p}-1}\left(K^{*} A^{p q-p} K+I-K^{*} K-D\right)}{p q-p}\right]: D>0\right\} \\
& =\max \left\{\operatorname{Tr}\left[C+\frac{C^{1-p q+p}\left(K^{*} A^{p q-p} K+I-K^{*} K-C^{p q-p}\right)}{p q-p}\right]: C=D^{\frac{1}{p q-p}}>0\right\} \\
& =\max \left\{\operatorname{Tr}\left[C\left(1-\frac{1}{p q-p}\right)+\frac{C^{1-p q+p} K^{*} A^{p q-p} K}{p q-p}+C^{1-p q+p}\left(I-K^{*} K\right)\right]: C>0\right\}
\end{aligned}
$$

Thus, the concavity of $F(A)$ is equivalent to the jointly concavity of $\operatorname{Tr}\left[\frac{C^{1-p q+p} K^{*} A^{p q-p} K}{p q-p}\right]$ for the strictly positive $A$ and $C$, which is the Lieb concavity theorem [22,23].

Unfortunately, Theorem 1 cannot be obtained using Epstein's theorem. Hence, we require a more general generalization of Epstein's theorem. First, for any $\operatorname{Im}(z)>0$, we know that $A+z B$ is invertible and $x^{*}(A+z B) x$ is a Pick function for any $x \in \mathbb{C}^{n}$ [14]. For any $A \in M(n, \mathbb{C})$, we know $f(A)$ is defined as [12]

$$
f(A)=\frac{1}{2 \pi} \oint_{C} \frac{f(z)}{z-A} \mathrm{~d} z,
$$

where $f(z)$ is a complex holomorphic function in an open set of the complex plane containing $\operatorname{Sp}(A)$ (the set of all eigenvalues of $A$ ). Then, we have the following lemma.

Lemma 4. Let $A, B \in H_{n}^{+}$and $0<\alpha \leq 1$, then

$$
x^{*}(A+z B)^{\alpha} x
$$

is a Pick function for any $x \in \mathbb{C}^{n}$ and $0<\arg \left(x^{*}(A+z B)^{\alpha} x\right)<\alpha \pi$ if $0<\arg (z)=\theta<\pi$, such as $\operatorname{Sp}\left((A+z B)^{\alpha}\right) \subseteq\left(\operatorname{Sp}(A+z B)^{\alpha}\right)$. Generally, we can find that

$$
x^{*} f(A+z B) x
$$

is a Pick function when $f$ is a Pick function.

Proof. Setting $z=\rho e^{i \theta}$, we have

$$
\begin{aligned}
(A+z B)^{\alpha} & =\int_{0}^{+\infty}\left(\frac{A+z B}{t+A+z B}\right) \mathrm{d} \mu(t) \\
& =\int_{0}^{+\infty}\left(\frac{1}{\frac{t}{A+z B}+1}\right) \mathrm{d} \mu(t),
\end{aligned}
$$

where $\mathrm{d} \mu(t)=\frac{t^{\alpha-1} \pi}{\sin \alpha \pi}$. 
Since $\operatorname{Im} z>0$, we see that $A+z B$ is invertible. Hence, we have

$$
\begin{aligned}
x^{*}(A+z B)^{\alpha} x & =\int_{0}^{+\infty} x^{*}\left(\frac{1}{\frac{t}{A+z B}+1}\right) x \mathrm{~d} \mu(t) \\
& =\int_{0}^{+\infty} y^{*}\left(\frac{t}{A+z^{*} B}+1\right) y \mathrm{~d} \mu(t), y=\left(\frac{t}{A+z B}+1\right)^{-1} x \\
& =\int_{0}^{+\infty} y^{*} y+t w^{*}(A+z B) w \mathrm{~d} \mu(t), w=\left(A+z^{*} B\right)^{-1} y \\
& =\int_{0}^{+\infty} y^{*} y+t w^{*} A w \mathrm{~d} \mu(t)+z \int_{0}^{+\infty} t w^{*} B w \mathrm{~d} \mu(t) .
\end{aligned}
$$

This implies that

$$
\operatorname{Im} x^{*}(A+z B)^{\alpha} x=\operatorname{Im}(z) \cdot \int_{0}^{+\infty} t w^{*} B w \mathrm{~d} \mu(t)>0
$$

hence, $0<\arg \left(x^{*}(A+z B)^{\alpha} x\right)$ when $0<\arg (z)=\theta<\pi$.

In the same way, we can obtain

$$
\operatorname{Im} w^{*}\left[\left(-A-z^{*} B\right)^{-\alpha}\right] w=\operatorname{Im}\left(e^{-i \alpha \pi} z^{*}\right) \cdot \int_{0}^{+\infty} t v^{*} B v \mathrm{~d} \mu(t)<0, v=\left(t\left(A+z^{*} B\right)+1\right)^{-1} w
$$

In particular, letting $w=\left(A+z^{*} B\right)^{\alpha} x$, we have

$$
\operatorname{Im}\left(e^{-i \alpha \pi} x^{*}(A+z B)^{\alpha} x\right)<0 .
$$

This is equivalent to $\arg \left(x^{*}(A+z B)^{\alpha} x\right)<\alpha \pi$.

To prove $\operatorname{Sp}\left((A+z B)^{\alpha}\right) \subseteq\left(\operatorname{Sp}(A+z B)^{\alpha}\right)$, let $(A+z B) \xi=\lambda \xi$, we find

$$
\xi^{*}(A+z B)^{\alpha} \xi=\left[\xi^{*}(A+z B) \xi\right]^{\alpha}=\left[\xi^{*} A \xi+z \xi^{*} B \xi\right]^{\alpha}=\rho^{\alpha} e^{i \alpha \theta}
$$

where $\tan \theta=\frac{\xi^{*} B \xi \operatorname{Im}(z)}{\xi^{*} A \xi^{\tau}+\zeta^{*} B \xi \operatorname{Re}(z)} \leq \tan \arg (z)$.

When $f(z)$ is a Pick function, using the integral represented of $f(z)$, in a similar way, we can obtain that

$$
x^{*} f(A+z B) x
$$

is a Pick function for any $x \in \mathbb{C}^{n}$.

Using Lemma 4, another proof of Theorem 1 can be obtained.

Theorem 2. For $0<p \leq 1,1<q \leq 2$ and $K^{*} K \leq I$, the function

$$
F(A)=\operatorname{Tr}\left[\exp _{q}^{\frac{1}{p}}\left(K^{*} \ln _{q}\left(A^{p}\right) K\right)\right]
$$

is concave for the strictly positive $A \in H_{n}^{+}$.

Proof. (The second proof of Theorem 1) 
First, setting $f(z)=\operatorname{Tr}\left[(A(z)+i B(z))^{\frac{1}{p q-p}}\right]$ where $A(z)=\operatorname{Re}\left(K^{*}(A+z B)^{p q-p} K+I-\right.$ $\left.K^{*} K\right)$ and $B(z)=\operatorname{Im}\left(K^{*}(A+z B)^{p q-p} K+I-K^{*} K\right) \in H_{n}^{+}$. As

$$
\begin{aligned}
\operatorname{Im}\left[\operatorname{Tr}\left[(A(z)+i B(z))^{\frac{1}{p q-p}}\right]\right] & =\operatorname{Im}\left[\operatorname{Tr}\left[\int_{0}^{+\infty}\left(\frac{A(z)+i B(z)}{t+A(z)+i B(z)}\right) \mathrm{d} \mu(t)\right]\right] \\
& =\operatorname{Im}\left[\int_{0}^{+\infty} \operatorname{Tr}\left[\left(\frac{\Lambda_{A(z)+i B(z)}}{t+\Lambda_{A(z)+i B(z)}} \mathrm{d} \mu(t)\right]\right]\right. \\
& =\operatorname{Im}\left[\int_{0}^{+\infty} \sum_{i=1}^{n}\left[\left(\frac{\lambda_{i}(A(z)+i B(z))}{t+\lambda_{i}(A(z)+i B(z))}\right) \mathrm{d} \mu(t)\right]\right] \\
& =\operatorname{Im}\left[\sum_{i=1}^{n}\left[\left(\lambda_{i}(A(z)+i B(z))^{\frac{1}{p q-p}}\right]\right]\right.
\end{aligned}
$$

when $\arg (z) \in(0, \pi)$ and $K^{*} K \leq I$, then

$$
\begin{aligned}
& \arg \left(\lambda_{i}(A(z)+i B(z))\right) \\
& =\arg \left(x_{i}^{*}(A(z)+i B(z)) x_{i}\right) \\
& =\arg \left(x_{i}^{*} K^{*}(A+z B)^{p q-p} K x_{i}+x_{i}^{*}\left(I-K^{*} K\right) x_{i}\right) \in(0,(p q-p) \pi),
\end{aligned}
$$

where $x_{i} \in \mathbb{C}^{n}$ are the eigenvectors of $K^{*}(A+z B)^{p q-p} K+I-K^{*} K$.

Hence,

$$
\operatorname{Im}\left[\operatorname{Tr}\left[(A(z)+i B(z))^{\frac{1}{p q-p}}\right]\right]=\operatorname{Im}\left[\sum_{i=1}^{n} z_{i}\right],
$$

where $z_{i}$ is the $i$ eigenvalue of $(A(z)+i B(z))^{\frac{1}{p q-p}}$ and $\arg \left(z_{i}\right) \in(0, \pi)$.

Thus, $f(z)=\operatorname{Tr}\left[(A(z)+i B(z))^{\frac{1}{p q-p}}\right]$ is a Pick function, and this implies that $F(A)$ is concave.

Using a similar method, we can obtain the following corollary.

Corollary 1. For $0<p \leq 1$ and $1<q \leq 2$, the function

$$
E(A)=\operatorname{Tr}\left[\exp _{q}^{\frac{1}{p}}\left[B+\ln _{q}\left(A^{p}\right)\right]\right]
$$

is concave for the strictly positive $A \in H_{n}^{+}$.

Since the Thompson-Golden theorem can be seen as a corollary of the Lieb concavity theorem, we discuss the Lieb concavity theorem for deformed exponentials. Setting $\mathrm{SP}(A) \subset\left\{z=\rho e^{i \theta}: 0<\rho, 0<\theta<\alpha\right\}$ and $\mathrm{SP}(B) \subset\left\{z=\rho e^{i \theta}: 0<\rho, 0<\theta<\beta\right\}$, then for any $A_{1}, B_{1} \in \mathrm{H}_{n}, A_{2}, B_{2} \in \mathrm{H}_{n}^{+}$and $A=A_{1}+i A_{2}, B=B_{1}+i B_{2}$, we have [12]

$$
\mathrm{SP}(A B) \subset\left\{z=\rho e^{i \theta}: 0<\rho, 0<\theta<\alpha+\beta\right\} .
$$

and then the following theorem can be obtained.

Theorem 3. For $0<p \leq 1,1<q \leq 2$ and $P^{*} P \leq I$, the following function

$$
L(A)=\operatorname{Tr}\left[\exp _{q}\left(P^{*} \ln _{q}\left(K^{*} A^{p} K\right) P\right) \exp _{q}\left(P^{*} \ln _{q} A^{1-p} P\right)\right]
$$

is concave for any $A \in H_{n}^{+}$.

Proof. Set $L_{A, B}(z)=\operatorname{Tr}\left[\exp _{q}\left(P^{*} \ln _{q}\left(K^{*}(A+z B)^{p} K\right) P\right) \exp _{q}\left(P^{*} \ln _{q}(A+z B)^{1-p} P\right)\right]$. 
When $x_{i} \in \mathbb{C}^{n}$ is a eigenvector of $P^{*}(A+z B)^{p q-p} P+I-P^{*} P$ and $P^{*} P \leq I$,

$$
\arg \left(x_{i}^{*} P^{*} K^{*}(A+z B)^{p q-p} K P x_{i}+x_{i}^{*}\left(I-P^{*} P\right) x_{i}\right) \in(0,(p q-p) \pi),
$$

if $\arg (z) \in(0, \pi)$. This implies

$$
\mathrm{SP}\left(P^{*} K^{*}(A+z B)^{p q-p} K P+I-P^{*} P\right) \subset\left\{z=\rho e^{i \theta}: 0<\rho, 0<\theta<(p q-p) \pi\right\},
$$

such as

$$
\mathrm{SP}\left(\exp _{q}\left(P^{*} \ln _{q}\left(K^{*}(A+z B)^{p} K\right) P\right)\right) \subset\left\{z=\rho e^{i \theta}: 0<\rho, 0<\theta<p \pi\right\} .
$$

Similarly, we can also obtain

$$
\mathrm{SP}\left(\exp _{q}\left(P^{*} \ln _{q}(A+z B)^{1-p} P\right)\right) \subset\left\{z=\rho e^{i \theta}: 0<\rho, 0<\theta<(1-p) \pi\right\} .
$$

Hence, using (8), we see that

$$
\begin{aligned}
& \mathrm{SP}\left[\exp _{q}\left(P^{*} \ln _{q}\left(K^{*}(A+z B)^{p} K\right) P\right) \exp _{q}\left(P^{*} \ln _{q}(A+z B)^{1-p} P\right)\right] \\
& \subset\left\{z=\rho e^{i \theta}: 0<\rho, 0<\theta<\pi\right\} .
\end{aligned}
$$

Thus, we know $\arg \left(L_{A, B}(z)\right) \in(0, \pi)$, which implies that $L_{A, B}(z)$ is a Pick function. Hence, $\mathrm{L}(\mathrm{A})$ is concave.

In fact, Theorem 3 is a generalization of the Lieb concavity theorem setting $P=I$, $K=\left(\begin{array}{cc}0 & 0 \\ H & 0\end{array}\right)$ and $A=\left(\begin{array}{cc}Z & 0 \\ 0 & B\end{array}\right)$. Moreover, we can obtain the following theorem.

Theorem 4. For $0<p, s \leq 1,1<q \leq 2$ and $P^{*} P \leq I$, the functions

$$
\operatorname{Tr}\left[\left[\exp _{q}\left(P^{*} \ln _{q} A^{\frac{p s}{2}} P\right) \exp _{q}\left(P^{*} \ln _{q}\left(K^{*} A^{s-s p} K\right) P\right) \exp _{q}\left(P^{*} \ln _{q} A^{\frac{p s}{2}} P\right)\right]^{\frac{1}{s}}\right]
$$

and

$$
\left[\operatorname{Tr} \exp _{q}\left(P^{*} \ln _{q} A^{\frac{p s}{2}} P\right) \exp _{q}\left(P^{*} \ln _{q}\left(K^{*} A^{s-s p} K\right) P\right) \exp _{q}\left(P^{*} \ln _{q} A^{\frac{p s}{2}} P\right)\right]^{\frac{1}{s}}
$$

are jointly concave for any $A \in H_{n}^{+}$.

The proof of Theorem 4 is similar to Theorem 3; here, we do not repeat the proof. In [19], Huang used exterior algebra to find that

$$
\left[\operatorname{Tr} \wedge^{k}\left[\exp \left(K^{*} \ln (A) K\right)\right]\right]^{\frac{1}{k}}
$$

is a concave function for any $A \in H_{n}^{+}, K^{*} K \leq I$ and $k \leq n$. Associated with Theorem 1, we can obtain a generalization as the following theorem.

Theorem 5. For $0<p \leq 1,1<q \leq 2$ and $K^{*} K \leq I$, the function

$$
\left[\operatorname{Tr} \wedge^{k}\left[\exp _{q}^{\frac{1}{p}}\left(K^{*} \ln _{q}\left(A^{p}\right) K\right)\right]\right]^{\frac{1}{k}}
$$

is concave for the strictly positive $A \in H_{n}^{+}$and $k \leq n$.

Proof. In fact, we can prove that

$$
\left[\operatorname{Tr} \wedge^{k}\left[\left(H^{*} A^{s} H+B\right)^{\frac{1}{s}}\right]\right]^{\frac{1}{k}}
$$


is a concave function for any $A \in H_{n}^{+}$where $0<s \leq 1$ and $B \in H_{n}^{+}$.

Using Theorem 1, we know that

$$
\operatorname{Tr}\left[\left(H^{*} A^{p} H+C\right)^{\frac{1}{p}}\right]
$$

is a concave function for any $A \in H_{n}^{+}$where $0<p \leq 1$ and $C \in H_{n}^{+}$.

Then, for any $A_{1}, A_{2} \in H_{n}^{+}$, we have

$$
\begin{aligned}
& {\left[\operatorname{Tr} \wedge^{k}\left[\left(H^{*}\left(\frac{A_{1}+A_{2}}{2}\right)^{s} H+B\right)^{\frac{1}{s}}\right]\right]^{\frac{1}{k}} } \\
= & {\left[\operatorname{Tr}\left[\left(\bar{H}^{*}\left(\frac{A_{1} \wedge^{k-1} I+A_{2} \wedge^{k} I}{2}\right)^{s} \bar{H}+\bar{B}\right)^{\frac{1}{s}}\right]\right]^{\frac{1}{k}} } \\
\geq & {\left[\operatorname{Tr}\left[\frac{\left(\bar{H}^{*}\left(A_{1} \wedge^{k-1} I\right)^{s} \bar{H}+\bar{B}\right)^{\frac{1}{s}}+\left(\bar{H}^{*}\left(A_{2} \wedge^{k-1} I\right)^{s} \bar{H}+\bar{B}\right)^{\frac{1}{s}}}{2}\right]\right]^{\frac{1}{k}} } \\
= & {\left[\operatorname{Tr}\left[\left(\frac{\left(H^{*} A_{1}^{s} H+B\right)^{\frac{1}{s}}+\left(H^{*} A_{2}^{s} H+B\right)^{\frac{1}{s}}}{2}\right) \wedge^{k-1}\left(H^{*}\left(\frac{A_{1}+A_{2}}{2}\right)^{s} H+B\right)^{\frac{1}{s}}\right]\right]^{\frac{1}{k}}, }
\end{aligned}
$$

where $\bar{H}=H \wedge^{k-1}\left(H^{*}\left(\frac{A_{1}+A_{2}}{2}\right)^{s} H+B\right)^{\frac{1}{s}}$ and $\bar{B}=B \wedge^{k-1}\left(H^{*}\left(\frac{A_{1}+A_{2}}{2}\right)^{s} H+B\right)^{\frac{1}{s}}$. Analogously, we can obtain

$$
\begin{aligned}
& {\left[\operatorname{Tr} \wedge^{k}\left[\left(H^{*}\left(\frac{A_{1}+A_{2}}{2}\right)^{s} H+B\right)^{\frac{1}{s}}\right]\right]^{\frac{1}{k}}} \\
& \geq\left[\operatorname{Tr}\left[\wedge^{k}\left(\frac{\left(H^{*} A_{1}^{s} H+B\right)^{\frac{1}{s}}+\left(H^{*} A_{2}^{s} H+B\right)^{\frac{1}{s}}}{2}\right)\right]\right]^{\frac{1}{k}} .
\end{aligned}
$$

Using lemma 3, we obtain

$$
\begin{aligned}
& {\left[\operatorname{Tr} \wedge^{k}\left[\left(H^{*}\left(\frac{A_{1}+A_{2}}{2}\right)^{s} H+B\right)^{\frac{1}{s}}\right]\right]^{\frac{1}{k}}} \\
& \geq \frac{\left[\operatorname{Tr}\left[\wedge^{k}\left(\left(H^{*} A_{1}^{s} H+B\right)^{\frac{1}{s}}\right)\right]\right]^{\frac{1}{k}}+\left[\operatorname{Tr}\left[\wedge^{k}\left(\left(H^{*} A_{2}^{s} H+B\right)^{\frac{1}{s}}\right)\right]\right]^{\frac{1}{k}}}{2} .
\end{aligned}
$$

Clearly, the proof of Theorem 5 is in the application of exterior algebra and the BrunnMinkowski inequality. Hence, other theorems, such as the Thompson-Golden theorem in a deformed exponential, can be generalized to a more general form, but we do not discuss this here.

\section{Conclusions}

In this paper, we used the Pick function to obtain a generalization of the Lieb concavity theorem and some corollaries. The advantage of using the Pick function is that it avoids discussing the commutativity of the matrix and variational method. Generally, we obtain that the following two functions are concave for $0<p, s \leq 1,1<q \leq 2$ and $P^{*} P \leq I$

$$
\left[\operatorname{Tr} \wedge^{k}\left[\exp _{q}\left(P^{*} \ln _{q} A^{\frac{p s}{2}} P\right) \exp _{q}\left(P^{*} \ln _{q}\left(K^{*} A^{s-s p} K\right) P\right) \exp _{q}\left(P^{*} \ln _{q} A^{\frac{p s}{2}} P\right)\right]^{\frac{1}{s}}\right]^{\frac{1}{k}}
$$


and

$$
\left[\operatorname{Tr} \wedge^{k}\left[\exp _{q}\left(P^{*} \ln _{q} A^{\frac{p s}{2}} P\right) \exp _{q}\left(P^{*} \ln _{q}\left(K^{*} A^{s-s p} K\right) P\right) \exp _{q}\left(P^{*} \ln _{q} A^{\frac{p s}{2}} P\right)\right]\right]^{\frac{1}{k s}},
$$

where $A \in H_{n}^{+}$and $k \leq n$, and this provides work for the future.

Author Contributions: Conceptualization, H.S.; writing-original draft, G.Y. and Y.L.; writingreview and editing, J.W.; funding acquisition, J.W. All authors have read and agreed to the published version of the manuscript.

Funding: The present research was supported by the General Project of Science and Technology Plan of Beijing Municipal Education Commission (Grant No. KM202010037003).

Institutional Review Board Statement: Not applicable.

Informed Consent Statement: Not applicable.

Data Availability Statement: Not applicable.

Acknowledgments: The authors thank the referees for detailed reading and comments that were both helpful and insightful.

Conflicts of Interest: The authors declare no conflict of interest.

\section{References}

1. Tropp, J.A. An introduction to matrix concentration inequalities. arXiv 2015, arXiv:1501.01571.

2. Petz, D. Quantum Information Theory and Quantum Statistics; Springer Science and Business Media: Berlin/Heidelberg, Germany, 2007.

3. Bellman, R. Introduction to Matrix Analysis; McGraw-Hill: New York, NY, USA, 1960.

4. Carlen, E. Trace inequalities and quantum entropy: An introductory course. Contemp. Math. 2010, 529, 73-140.

5. Zhang, F. Matrix Theory: Basic Results and Techniques; Springer: Berlin/Heidelberg, Germany, 1999.

6. Simon, B. Trace Ideals and Their Applications. In Mathematical Surveys and Monographs, 2nd ed.; American Mathematical Society: Providence, Rhode Island, 2005.

7. Wigner, E.P.; Yanase, M.M. On the positive definite nature of certain matrix expressions. Cunud. J. Math. 1964, 16, 397-406. [CrossRef]

8. Lieb, E. Convex trace functions and the Wigner-Yanase-Dyson conjecture. Adv. Math. 1973, 11, 267-288. [CrossRef]

9. $\quad$ Bhatia, R. Positive Definite Matrices; Princeton University Press: Princeton, NJ, USA, 2007.

10. Ando, T. Concavity of certain maps on positive definite matrices and applications to Hadamard products. Linear Algebra Appl. 1979, 26, 203-241. [CrossRef]

11. Effros, E.G. A matrix convexity approach to some celebrated quantum inequalities. Proc. Natl. Acad. Sci. USA 2009, 106, 1006-1008. [CrossRef] [PubMed]

12. Epstein, H. Remarks on two theorems of E. Lieb. Comm. Math. Phys. 1973, 31, 317-325. [CrossRef]

13. Shi, G.H.; Hansen, F. Variational representations related to Tsallis relative entropy. Lett. Math. Phys. 2020, 110, 2203-2220. [CrossRef]

14. Donogue, W. Monotone Matrix Functions and Analytic Continuution; Springer: New York, NY, USA, 1974.

15. Davis, C. Notions generalizing convexity for functions defined on spaces of matrices. In Convexity: Proceedings of the Seventh Symposium in Pure Mathematics of the American Mathematical Society; American Mathematical Society: Providence, Rhode Island, 1963; pp. 187-201.

16. Choi, M.D. A Schwarz inequality for positive linear maps on $C^{\star}$-algebras. Illinois J. Math. 1974, 18, 565-574. [CrossRef]

17. Bendat, J.; Sherman, S. Monotone and convex operator functions. Trans. Am. Math. Soc. 1955, 79, 58-71. [CrossRef]

18. Hansen, F. Trace Functions with Applications in Quantum Physics. J. Stat. Phys. 2014, 154, 807-818. [CrossRef]

19. Huang, D. A generalized Lieb's theorem and its applications to spectrum estimates for a sum of random matrices. Linear Algebra Appl. 2019, 579, 419-448. [CrossRef]

20. Marshall, A.W.; Olkin, I.; Arnold, B.C. Inequalities: Theory of Majorization and Its Applications; Springer: New York, NY, USA, 2011.

21. Bhatia, R. Matrix Analysis; Springer: Berlin/Heidelberg, Germany, 1997.

22. Aujla, J.S. A simple proof of Lieb concavity theorem. J. Math. Phys. 2011, 52, 043505. [CrossRef]

23. Nikoufar, I.; Ebadian, A.; Gordji, M.E. The simplest proof of Lieb concavity theorem. Adv. Math. 2013, 248, 531-533. [CrossRef] 